\title{
Direct observation of chaotic resonances in optical microcavities
}

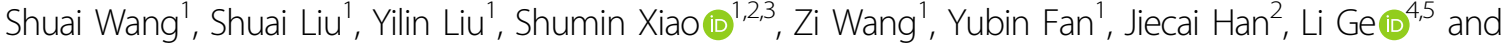 \\ Qinghai Song (DD ${ }^{1,3}$
}

\begin{abstract}
Optical microcavities play a significant role in the study of classical and quantum chaos. To date, most experimental explorations of their internal wave dynamics have focused on the properties of their inputs and outputs, without directly interrogating the dynamics and the associated mode patterns inside. As a result, this key information is rarely retrieved with certainty, which significantly restricts the verification and understanding of the actual chaotic motion. Here we demonstrate a simple and robust approach to directly and rapidly map the internal mode patterns in chaotic microcavities. By introducing a local index perturbation through a pump laser, we report a spectral response of optical microcavities that is proportional to the internal field distribution. With this technique, chaotic modes with staggered mode spacings can be distinguished. Consequently, a complete chaos assisted tunneling (CAT) and its time-reversed process are experimentally verified in the optical domain with unprecedented certainty.
\end{abstract}

\section{Introduction}

The detailed understanding of mixed-phase space with both regular and chaotic motions is essential for a wide range of physical systems, ranging from atomic and molecular physics to mesoscopic science and even astrophysics ${ }^{1-3}$. Among the cornucopia of quantum chaotic phenomena in mixed-phase space, the chaos assisted tunneling (CAT) between distinct regular phase space regions separated by a chaotic sea is particularly interesting and has been intensively studied in molecular dynamics, cold atom assemblies, and whispering gallery modes (WGM) microcavities ${ }^{4-7}$. Among these systems, WGM microcavities have a series of advantages to study CAT, including easy control of internal quantum or classical dynamics and multiple manifestations of CAT

\footnotetext{
Correspondence: Shumin Xiao (shumin.xiao@hit.edu.cn) or Li Ge (li.ge@csi. cuny.edu) or Qinghai Song (qinghai.song@hit.edu.cn)

${ }^{1}$ Ministry of Industry and Information Technology Key Lab of Micro-Nano Optoelectronic Information System, Harbin Institute of Technology (Shenzhen), Shenzhen 518055, China

${ }^{2}$ National Key Laboratory of Science and Technology on Advanced Composites in Special Environments, Harbin Institute of Technology, Harbin 150080, China Full list of author information is available at the end of the article

These authors contributed equally: Shuai Wang, Shuai Liu
}

beyond the regular level splitting ${ }^{7-19}$. In past decades, the rapid progress in WGM microcavities ${ }^{20-22}$ has not only enriched fundamental physics such as CAT, resonance assisted tunneling ${ }^{23}$, and turnstile transport ${ }^{24}$, but also enabled many practical applications from broadband coupling $^{16,25,26}$ to integrated laser sources ${ }^{27-29}$, sensors $^{30,31}$ and all-optical switches ${ }^{32}$.

Despite these achievements, the conventional study of mixed-phase space in optical WGM microcavities focuses on its ancillary signatures in the inputs and the outputs, without directly interrogating the dynamics and the associated mode patterns inside. These techniques include, for example, comparing the experimentally observed free spectral range (FSR) and far-field radiation patterns with theoretical predictions ${ }^{11,12,18,19,33-36}$. However, such comparisons often lead to educated conjectures instead of conclusive affirmation, especially when multiple mode groups have similar FSRs and far-field patterns ${ }^{37,38}$. The association of internal mode patterns with experimentally observed resonances is hence crucial to provide a clearer picture of quantum chaos in optical microcavities.

\section{(c) The Author(s) 2021}

(c) (i) Open Access This article is licensed under a Creative Commons Attribution 4.0 International License, which permits use, sharing, adaptation, distribution and reproduction cc) in any medium or format, as long as you give appropriate credit to the original author(s) and the source, provide a link to the Creative Commons license, and indicate if changes were made. The images or other third party material in this article are included in the article's Creative Commons license, unless indicated otherwise in a credit line to the material. If material is not included in the article's Creative Commons license and your intended use is not permitted by statutory regulation or exceeds the permitted use, you will need to obtain permission directly from the copyright holder. To view a copy of this license, visit http://creativecommons.org/licenses/by/4.0/. 
Inspired by a technique developed in the microwave domain $^{9,39,40}$, here we propose and demonstrate a simple, robust, and contactless mechanism to rapidly map the field patterns in silicon microdisks. Compared with other attempts ${ }^{10,41,42}$ at visualizing optical modes inside a cavity, our method allows the observation of modes with drastically different dynamics, covering phase space structures such as unbroken Kolmogorov-Arnold-Moser (KAM) curves, stable periodic motions, and the chaotic sea. Using this technique, we report the observation of the complete process of CAT and its time-reversed counterpart with unprecedented assurance in the optical domain.

\section{Results}

\section{Field mapping principle}

Figure 1 shows the schematic of the working principle. The setup utilizes the conventional measurement scheme for on-chip integrated silicon microdisks (see Supplementary Section 1), with the crucial addition of a

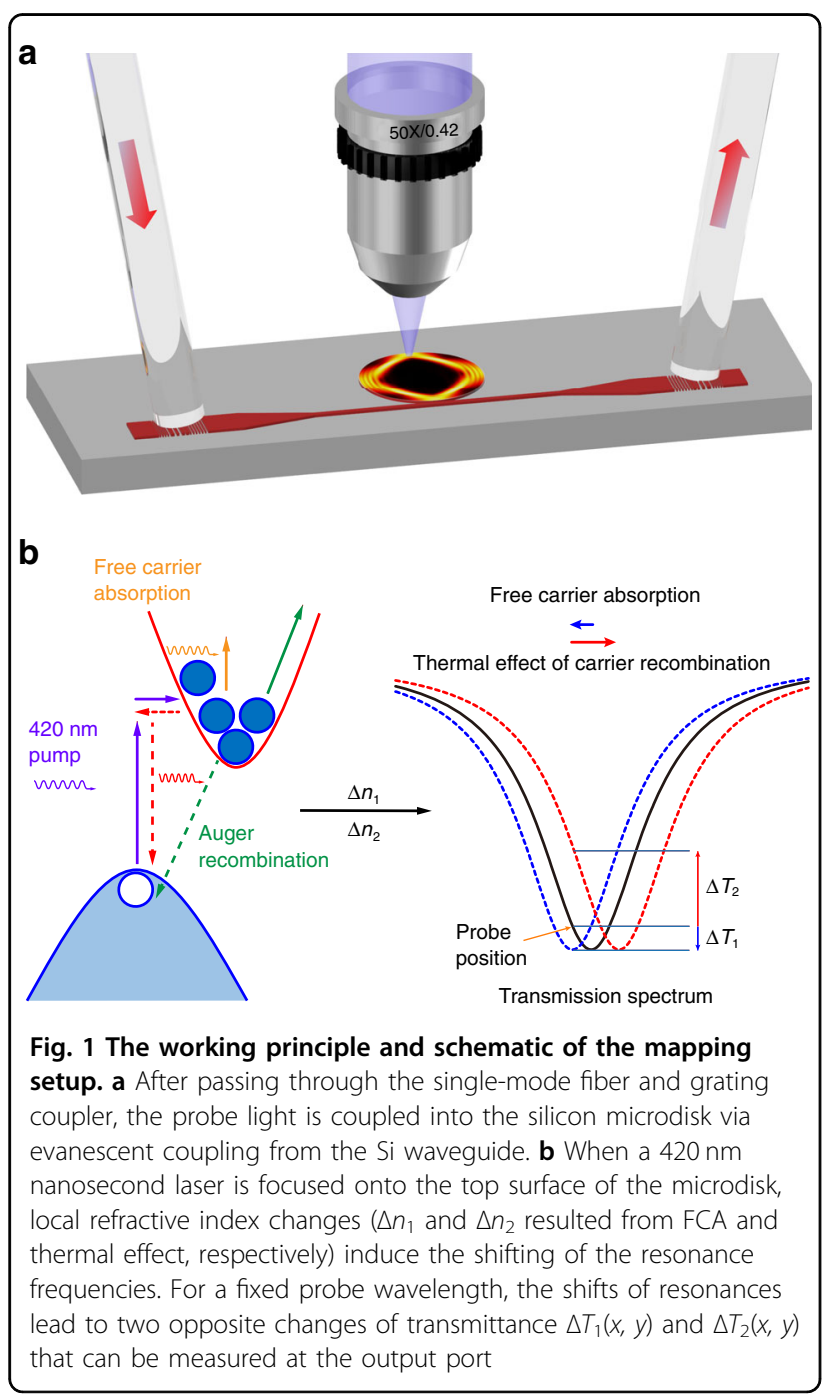

nanosecond laser at $420 \mathrm{~nm}(6 \mathrm{~ns}$ pulse duration, $10 \mathrm{~Hz}$ repetition rate) normally focused on the microdisk through a $50 \times$ objective lens $(\mathrm{NA}=0.42)$. The probe laser in the single-mode fiber is tuned to the shorter wavelength side of each resonance. The above-bandgap optical excitation induces free-carrier absorption (FCA) as well as thermal heating within the silicon, both of which lead to induced local changes of the refractive index by $\Delta n_{1}$ and $\Delta n_{2}{ }^{41,43}$, respectively. For the range of pump laser in our experiments, we estimate that $\Delta n$ is on the order of 0.01 or smaller. The mode patterns in our system are only minutely perturbed and keep their geometric character, e.g., as a WGM or localized on a periodic orbit (see Supplementary Section 2). Correspondingly, the resonant wavelengths shift, following the equation

$$
\Delta \lambda\left(x_{0}, y_{0}\right)=\lambda_{0} \int \frac{\Delta n(x, y ; x 0, y 0)}{n} \psi_{0}^{2}(x, y) d x d y
$$

in the absence of strong mode mixing ${ }^{44}$. Here $\lambda_{0}$ is the unperturbed resonant wavelength, $n$ is the unperturbed cavity refractive index, a constant for our optical microcavities. $\Delta n\left(x, y ; x_{0}, y_{0}\right)$ is its change due to the pump beam centered at $\left(x_{0}, y_{0}\right)$, and $\psi_{0}(x, y)$ is the corresponding out-of-plane $H$ field normalized by $\int \psi_{0}^{2}(x, y) d x d y=1$. For a fixed value of $\Delta n$, Eq. (1) shows clearly that the wavelength shift is proportional to the field distribution (i.e., $\left.\psi_{0}^{2}(x, y)\right)$ at the pump spot. As a result, by performing a two-dimensional scan on the surface of the microdisk with a fixed pump density, the field pattern inside can be read out from the corresponding wavelength shift in the transmission spectrum. However, instead of measuring this wavelength shift directly, an easier approach is to measure the change of the transmission at a fixed wavelength, set by the probe laser close to each unperturbed resonance (See the discussion on the influence of the probe wavelength in Supplementary Section 2). Here we note that $\Delta n_{1}$ and $\Delta n_{2}$ due to FCA and thermal heating have opposite signs, and so are the resulting changes of the transmission (denoted by $\Delta T_{1}\left(x_{0}, y_{0}\right)$ and $\left.\Delta T_{2}\left(x_{0}, y_{0}\right)\right)$ in Fig. $1 \mathrm{~b}$. Since the former is on the order of noise in our experiment, only the latter is extracted to represent the field distribution (see detailed discussion in Supplementary Section 3).

\section{Verification in circular microcavity}

To validate the proposed measurement scheme, we apply it to a circular silicon microdisk fabricated on a $220 \mathrm{~nm}$ silicon on insulator (SOI) wafer with a combined process of electron beam lithography and reactive ion etching (see "Methods"). Figure 2a shows the top-view scanning electron microscope (SEM) image of the silicon microdisk. It is a circular disk with $R=5 \mu \mathrm{m}$ coupled to a 
a
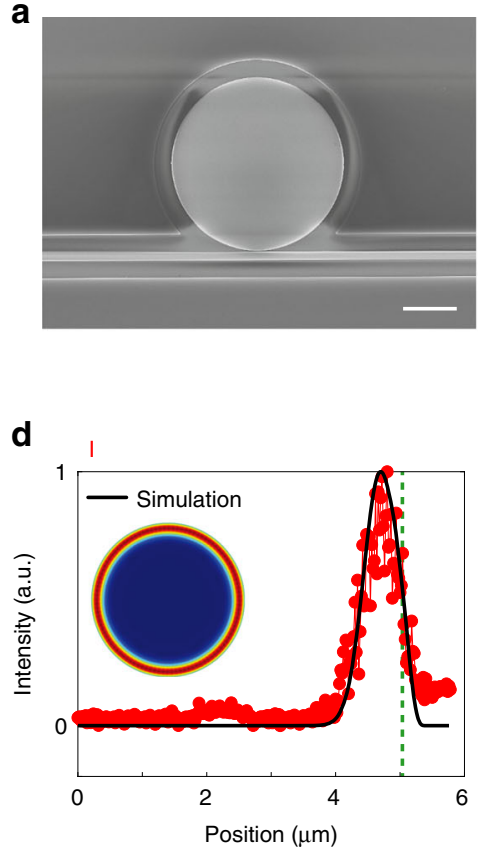

b

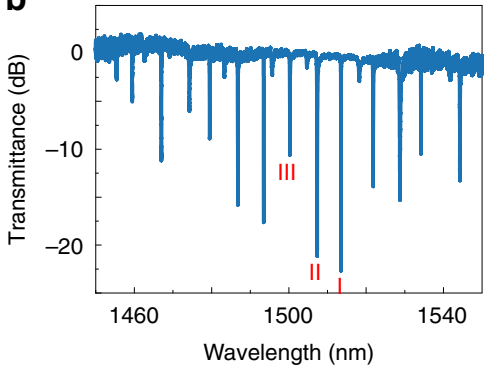

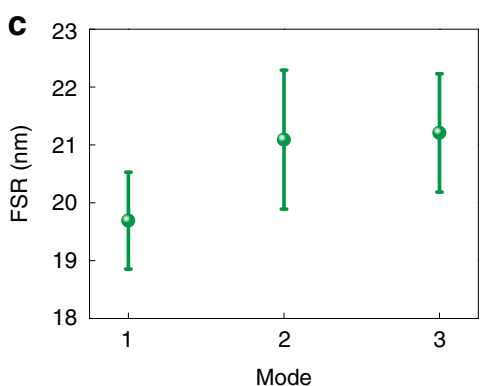
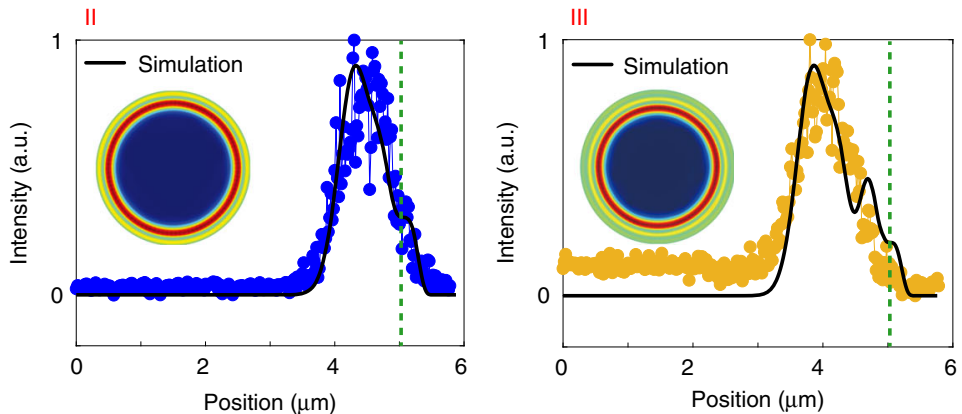

Fig. 2 Validation of the proposed method in a circular microdisk. a. Top-view SEM image of a silicon microdisk. The radius of the microdisk is $5 \mu \mathrm{m}$, the widths of the bus waveguide and the gap are $458 \mathrm{~nm}$ and $80 \mathrm{~nm}$, respectively. The scale bar is $3 \mu \mathrm{m}$. $\mathbf{b}$. Experimentally recorded transmission spectrum along the bus waveguide. $\mathbf{c}$. Average FSR of three sets of resonances in the silicon microdisk. $\mathbf{d}$. Experimentally recorded field distributions of resonances I, II, III in Fig. 2b. Here only the intensity along the radius is considered due to the rotational symmetry. Scan along the horizontal axis, starting from the center of the cavity to the right edge. The numerically calculated field distributions after taking the $600 \mathrm{~nm}$ laser spot size into account are shown as solid lines for a direct comparison. The insets are the corresponding numerically calculated traveling wave field patterns

bus waveguide. The transmission spectrum is recorded at the throughput of the bus waveguide and plotted in Fig. 2b. Different from an active microlaser with an embedded gain medium, here a large number of resonances with different quality $(Q)$ factors can be resolved in this passive microdisk. Taking the modes marked by I, II, III in Fig. 2b as an example, the FWHMs are $0.110 \mathrm{~nm}, 0.305 \mathrm{~nm}$, $0.145 \mathrm{~nm}$ and the corresponding $Q$ factors are 13759, 4950, and 10347, respectively (see details in Supplementary Section 8). It was commonly hypothesized that the field distribution of a particular WGM can be obtained by analyzing its FSR and $Q$ factor. However, such an approach is at most an educated conjecture in nanofabricated systems, where the shape of the microdisk deviates minutely from the designed one. For example, using a simple estimation of the FSR given by $\lambda^{2} / 2 \pi n_{\text {group }} R$, the most prominent resonances in Fig. $2 \mathrm{~b}$ can be categorized into three groups. As shown in Fig. 2c, their respective FSRs are very close and hence cannot be used reliably to deduce the spatial features of the corresponding WGMs; their $Q$ factors do not display a systematic difference, either.

In contrast, our method based on the local index variation gives convincing and clearly distinct results. For mode I to III, one from each group mentioned earlier. We are able to map their field distributions along the cavity radius with the setup in Fig. 1a. The pump density is fixed at $1 \mathrm{~nJ}\left(\mu \mathrm{m}^{2}\right)^{-1}$, and the probe laser is tuned to $1513.489 \mathrm{~nm}, 1507.331 \mathrm{~nm}$, and $1500.258 \mathrm{~nm}$ for three modes, respectively. The experimental results are summarized using connected dots of different colors in Fig. $2 \mathrm{~d}$, from which it is clear that modes I-III are most intense at $0.33 \mu \mathrm{m}, 0.8 \mu \mathrm{m}$, and $1.14 \mu \mathrm{m}$ away from the disk boundary (green dashed lines), respectively. In the meanwhile, we also observe the increase of the full width at half maximum (FWHM) of the field distribution from mode I to III. These features are consistent with the simulated WGMs after taking the focal spot size into account (solid lines in Fig. 2d), indicating that the three aforementioned groups of resonances have radial quantum numbers of $j=1,2,3$, respectively, which cannot be retrieved indisputably using conventional methods.

In our experiment, the laser spot size is around $600 \mathrm{~nm}$ (see Supplementary Section 6), which depends on the pump wavelength and the NA of objective lens. Considering the diffusion of thermal heating effect, the final resolution is determined by the laser spot size and thermal diffusivity in silicon (see details in Supplementary Section 

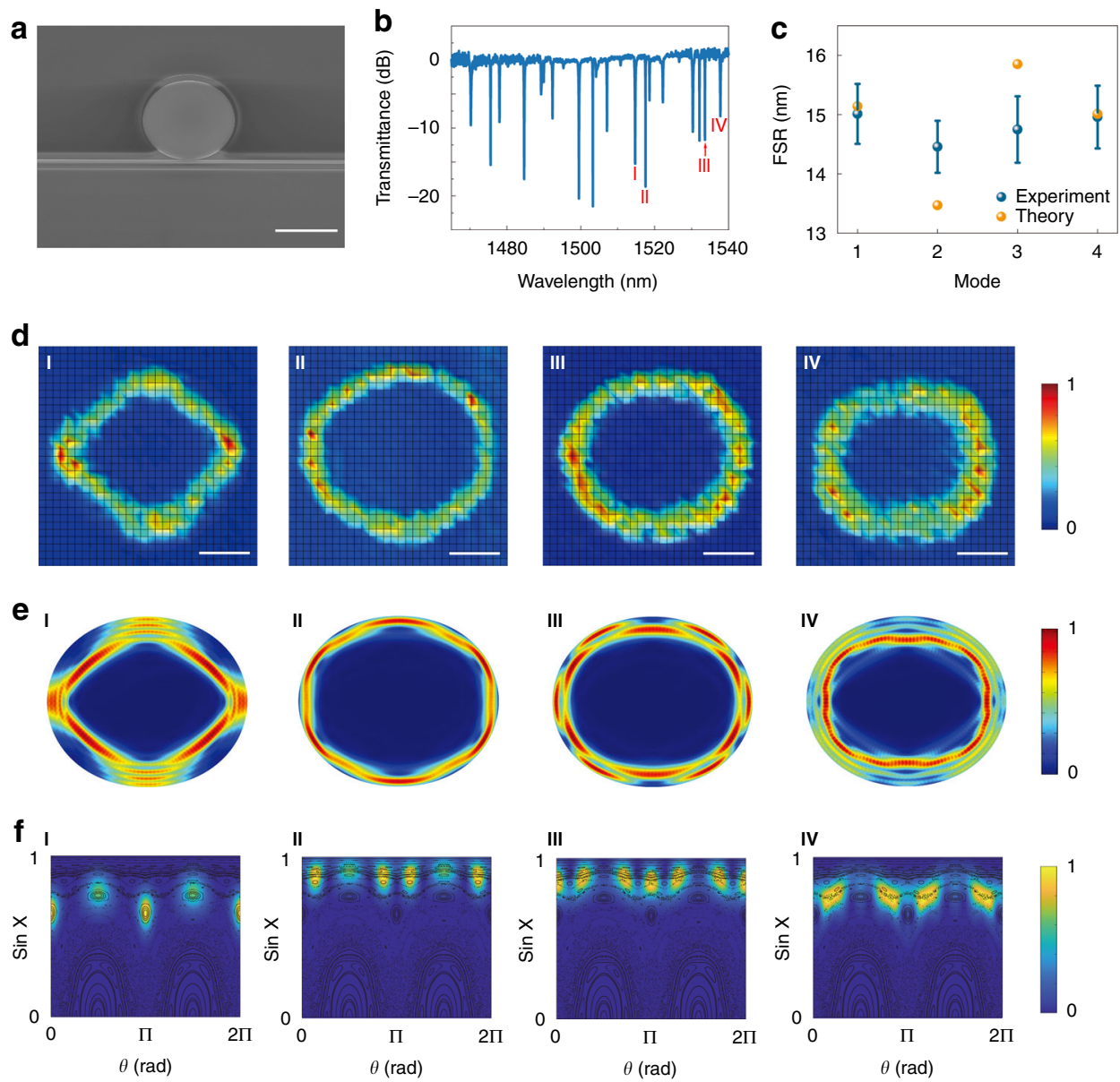

Fig. 3 Mapping resonances in the deformed silicon microdisk. a Top-view SEM image of the quadrupolar microdisk. Here $R=7 \mu \mathrm{m}$ and $\varepsilon=0.08$. The width of bus waveguide and the gap size are $462 \mathrm{~nm}$ and $80 \mathrm{~nm}$, respectively. The scale bar is $10 \mu \mathrm{m}$. $\mathbf{b}$ Experimentally recorded transmission spectrum along the bus waveguide. c Mode spacings of four groups of high $Q$ resonances. Orange dots show their analytical values in an ideal quadruple. $\mathbf{d}$ Experimentally mapped field distributions of mode I-IV. The scale bars are $4 \mu \mathrm{m}$. $\mathbf{e}$ and $\mathbf{f}$ the corresponding numerically calculated traveling wave mode patterns and their Husimi maps

6). While the transverse structures of propagation mode can be resolved, the laser spot size can still be improved to 200-300 $\mathrm{nm}$ by employing shorter pump wavelength, larger NA objective lens, and high-speed photodetector. The scanning speed can also be further increased with the assistance of Galvo scanning mirror (see details in Supplementary Section 6).

\section{Mapping different field patterns in one deformed microcavity}

Assured by these definitive findings, we proceed to consider the quadruple cavity and its CAT process. The cavity shape is defined by $\rho(\theta)=R(1+\varepsilon \cos 2 \theta)$ in polar coordinates, and the classical ray dynamics become increasingly chaotic as the deformation parameter $\varepsilon$ becomes larger ${ }^{7}$. An example of the Poincaré surface of section (PSOS) at $\varepsilon=0.08$ is given in the in the Supplementary Section 4, showing a mixture of regular and chaotic regions. It consists of unbroken KAM curves close to $\sin X=1$, representing the quasi WGMs. At intermediate $\sin X \sim[0.4,0.9]$, there are islands corresponding to stable periodic motions, which are surrounded by the chaotic regions in the PSOS and above the critical angle $X_{c}=\sin ^{-1}(1 / n)$ in silicon. As such, this quadruple microdisk, fabricated with the same process as above (see Fig. 3a), provides an ideal platform for the study of CAT and other chaotic motions.

Due to the complex phase space structures, multiple resonances with similar high $Q$ factors exist that are confined by total internal reflection along stable periodic orbits or by wave localization in the chaotic sea, leading to a more complicated transmission spectrum (see Fig. 3b) compared with the circular disk. Nevertheless, four groups of modes can be separated by estimating the FSRs of periodic orbits again. As depicted in Fig. 3c, their FSRs are even closer than the circular disks and strongly 
overlap, and it is extremely challenging to identify the modes confined on stable islands with certainty. Even if the far-field angular distributions of these resonances are measured with an infrared camera, to associate them with a particular CAT process in the quadruple microdisk is still merely an assumption. This is because that the regular modes within stable islands usually have similar farfield emissions as their chaotic counterparts.

The above difficulty can be solved by mapping the field patterns with the setup introduced in Fig. 1a. Modes I-IV, one from each of the four groups, are selected and experimentally studied. Here the pump laser scans 33 steps in both the horizontal and vertical directions, totaling 1089 locations on the entire top surface of the quadruple cavity. For the resonance at $1514.715 \mathrm{~nm}$ (mode I), the field pattern is obtained by plotting the change of transmittance $\Delta T$ as a function of position $(x$, $y$ ). As depicted in Fig. 3d-I, the field pattern shows a clear four-bounce diamond shape. Compared with the numerical results (Fig. 3e-I) and the Husimi map (Fig. $3 \mathrm{f}-\mathrm{I}$ ), we can confirm that the resonances in group-I are confined within the period-4 stable islands in $\sin X \sim[0.6$, $0.8]$. By changing the probe wavelength to $1517.523 \mathrm{~nm}$ (mode II), $1533.596 \mathrm{~nm}$ (mode III), $1537.78 \mathrm{~nm}$ (mode IV), the corresponding field patterns have also been mapped and shown in Fig. 3d-II to Fig. 3d-IV. By comparing the experimental observations and their autocorrelation (see Supplementary Section 7) with the field patterns and Husimi maps, we can identify them as a stable six-bounce mode (Fig. 3e-II and Fig. 3f-II), a scar mode on the unstable six-bound orbit (Fig. 3e-III and Fig. $3 \mathrm{f}-\mathrm{III}$ ), and a scar mode on the unstable rectangular orbit (Fig. 3e-IV and Fig. 3f-IV). The analytical FSRs of these mode groups are shown in Fig. 3c, and their deviations from the experimental data reiterate the limitation of judging modes solely by their FSRs.

\section{Experimental confirmation of CAT}

The ability to distinguish different resonances with similar FSRs demonstrated above is essential in the study of the CAT process. Figure 4a shows the microscope and top-view SEM images of a quadruple cavity of the same design, except for the addition of a channeling waveguide $^{18}$ connected at $\theta=-36.3^{\circ}$. Light in many resonances can escape from this channeling waveguide (see the top panel in Fig. 4b), and the transmission peaks collected in this waveguide can also be separated into several groups with nearly identical FSRs. It is important to note that the majority of them are due to direct transport, which takes place when the channeling waveguide overlaps with the phase space structure of a mode in the PSOS, including modes II-IV studied in Fig. 3. Therefore, it is impossible to single out transmission peaks associated with CAT just based on the FSR.
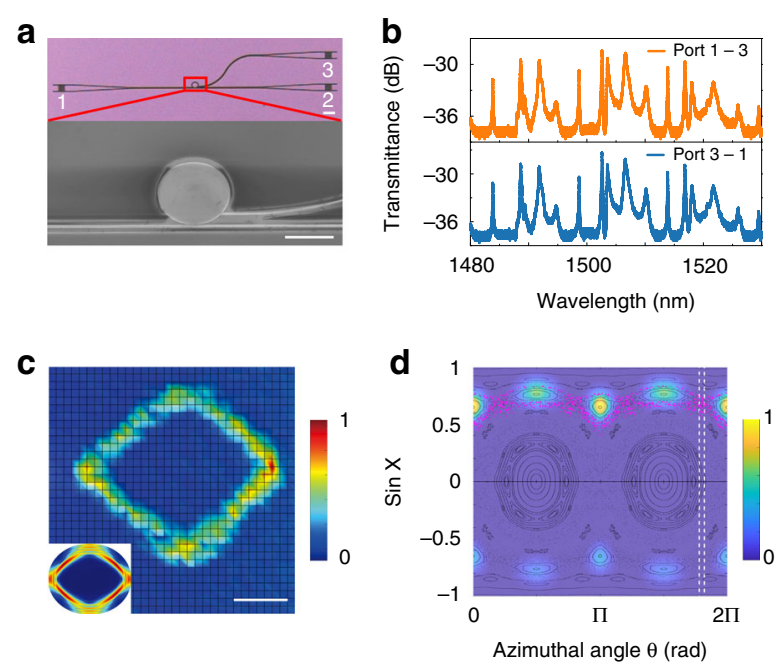

Fig. 4 Direct observation of CAT. a Microscopic and top-view SEM images of the silicon microdisk with a channeling waveguide of width $445 \mathrm{~nm}$. The scale bar is $10 \mu \mathrm{m}$. b Top: Transmission spectrum from the bus waveguide (port-1) to the channeling waveguide (port-3). Bottom: the same but from port-3 to port-1. c Correspondence of experimentally recorded field pattern and theoretically calculated mode pattern at $\lambda=1498.66 \mathrm{~nm}$. The scale bar is $4 \mu \mathrm{m}$. $\mathbf{d}$ The Husimi map of the resonance at $\lambda=1498 \mathrm{~nm}$. The ray dynamics are overlaid for a direct comparison, with the pink dots showing rays tunneled from the period-4 islands to the chaotic sea. The vertical box of white dashed line represents the leaky channel of the channeling waveguide

Fortunately, the diamond modes are confined in the period-4 islands, which are clearly separated from the waveguide in the PSOS (see vertical box of white dashed lines in Fig. 4d). As a result, light in these modes can only escape via CAT and then be collected by the channeling waveguide at port-3. Therefore, the existence of CAT can be verified by observing a transmission peak in the channeling waveguide at the wavelength of a diamond mode determined by our method. One such confirmed CAT-induced transmission peak in Fig. $4 \mathrm{~b}$ is at $1498.66 \mathrm{~nm}$, and its clear diamond shape is shown in Fig. 4c (see transmission spectrum along the bus waveguide in Supplementary Section 9). This is the first time that a complete process including the evanescent coupling to modes in stable islands, the CAT, and the collection via channeling waveguide has been experimentally confirmed.

\section{The time-reversal process of CAT}

The quadrupole microdisk in Fig. 4 is a timeindependent system, described by a symmetric electric permittivity tensor $(\varepsilon)$ and a symmetric magnetic permeability tensor $(\mu)$. In principle, this system should be constrained by the Lorentz reciprocity theorem ${ }^{45-47}$. Once the chaos is taken into account, the situation 
becomes totally different. The presence of chaos introduces inherent one-way stable structures in phase space. While individual trajectories are time-reversal, the averages in an experimental measurement invariably lead to (chaotic) attractor in the forward time direction, and the (chaotic) repeller in the reversed time direction ${ }^{3,48}$. Consequently, the CAT in optical microcavities is tend to be considered as an irreversible process ${ }^{49}$.

With the above technique, we are able to experimentally explore the time-reversal process of CAT for the first time. In this experiment, the experimental setup and the device are the same as the CAT experiment except that the probe light is sent in port- 3 and the transmission spectrum is detected in port-1. The experimental results are plotted in the bottom panel of Fig. 4b. We observe again the transmission peak at $1498.66 \mathrm{~nm}$ with the same diamond mode. The backward transmittance is almost the same as the forward one. In fact, the entire transmission spectrum is very close to before (top panel of Fig. 4b) within measurement precision, providing strong experimental evidence of optical reciprocity ${ }^{45,46}$ involving CAT.

It is obvious that our experimental observation is in contradiction to the conventional belief of nonreciprocity in a chaotic system. This difference comes from our design. As depicted in Fig. 4d, the channeling waveguide introduces a new leaky channel marked by white dashed lines. The forward transmission is determined by the overlapping between chaotic layer surrounding the stable islands (pink dots) and the vertical box, which is far above the critical line and happens earlier than the refractive transmission ${ }^{18,50}$. Because port- 3 is a single-mode waveguide, it can only collect a tiny fraction of the states with incident angles almost parallel to the waveguide. Such a small angular divergence is not sufficient to cause any observable breakdown of reciprocity in chaotic cavities.

\section{Discussion}

In summary, we have demonstrated a powerful approach that reveals the internal field patterns of optical modes with drastically different dynamics, covering phase space structures such as unbroken KAM curves, stable periodic orbits, and the chaotic sea. This technique has been applied to confirm the CAT process with unprecedented assurance. Meanwhile, in contrast to conventional belief of chaotic system, the time-reversal process of CAT has also been verified for the first time. We also note that the main setup is similar to laser direct writing. Therefore, the in-plane laser spot size can be further improved to around $200 \mathrm{~nm}$ by optimizing the system with twophoton absorption and pulse duration (see Supplementary Section 6). The same technique can also be extended to active devices $^{51,52}$ as well. We expect this research to offer new possibilities in the understanding of physical processes in optical microcavities and maximize the light-matter interactions within ${ }^{53,54}$.

\section{Materials and methods \\ Numerical simulation}

The resonances within the microdisks are calculated using the finite element method (COMSOL Multiphysics 5.4). We use a two-dimensional model with an effective index, and an outgoing boundary condition is implemented by perfectly matched layers applied outside the cavity. The eigenfrequencies $(\omega)$ of the resonances within the microdisks are then calculated with the photonics module, and their $Q$ factors are derived using $Q=\operatorname{Re}(\omega) /|2 \operatorname{Im}(\omega)|$. The wavefunctions on the cavity boundaries are also extracted to produce the Husimi map, obtained by projecting the wavefunctions to the phase space via the Husimi Functions.

\section{Device fabrication}

The devices are fabricated on a SOI wafer with $220 \mathrm{~nm}$ top silicon device layer on $3 \mu \mathrm{m}$ buried oxide. Then $340 \mathrm{~nm}$ photo resist ZEP $520 \mathrm{~A}$ is spin-coated onto the wafer as the soft mask. The silicon microdisks are patterned by the electron beam lithography (Raith E-Line) with $30 \mathrm{kV}$, then the sample was fully etched by using reactive ion etcher (Oxford, RIE80) (see details in Supplementary Section 1).

\section{Optical measurements}

The incident light as a probe laser in the single-mode fiber is slightly tuned to the shorter side of the resonant wavelength and coupled into microdisk. Then the $420 \mathrm{~nm}$ nanosecond pump laser is focused on sample top surface through a $50 \times$ objective lens $(\mathrm{NA}=0.42)$, which is fixed on an electric three-dimensional translation stage. The focal spot is about $610 \mathrm{~nm}$, which is diffraction limited (see details in Supplementary Section 1).

\section{Acknowledgements \\ The authors thank H. Cao and A. D. Stone for useful discussions and suggestions. The authors acknowledge the financial support from National Natural Science Foundation of China under Grant No. 2018YFB2200400, the National Science Foundation (JCYJ20180306172041577 NSF (61975041), JCYJ20180306172041577 NSF (11974092)), and the Shenzhen Fundamental research projects (JCYJ20180507183532343, JCYJ20180507184613841).}

\section{Author details \\ ${ }^{1}$ Ministry of Industry and Information Technology Key Lab of Micro-Nano Optoelectronic Information System, Harbin Institute of Technology (Shenzhen), Shenzhen 518055, China. ${ }^{2}$ National Key Laboratory of Science and Technology on Advanced Composites in Special Environments, Harbin Institute of Technology, Harbin 150080, China. ${ }^{3}$ Collaborative Innovation Center of Extreme Optics, Shanxi University, Taiyuan 030006, China. ${ }^{4}$ Department of Physics and Astronomy, College of Staten Island, CUNY, Staten Island, NY 10314, USA. ${ }^{5}$ The Graduate Center, CUNY, New York, NY 10016, USA}

\section{Author contributions}

Q.S., S.X., L.G. designed the experiments. S.W., Y.L. fabricated the micro-devices. S.W., S.L., Z.W., Y.F. performed the optical characterizations. S.W., S.L. did the 
numerical calculations. All the authors analyzed the data. Q.S., S.X., J.H., L.G. wrote the manuscript.

\section{Data availability}

All data needed to evaluate the conclusions in the paper are present in the paper and/or the Supplementary Materials. Additional data related to this paper may be requested from the authors.

\section{Conflict of interest}

The authors declare no competing interests.

Supplementary information The online version contains supplementary material available at https://doi.org/10.1038/s41377-021-00578-7.

Received: 15 April 2021 Revised: 15 June 2021 Accepted: 17 June 2021 Published online: 30 June 2021

\section{References}

1. Keshavamurthy, S. \& Schlagheck, P. Dynamical Tunneling: Theory and Experiment. (Boca Raton: CRC Press, 2011).

2. Davis, M. J. \& Heller, E. J. Quantum dynamical tunneling in bound states. J. Chem. Phys. 75, 246-254 (1981).

3. Cao, H. \& Wiersig, J. Dielectric microcavities: model systems for wave chaos and non-hermitian physics. Rev. Mod. Phys. 87, 61-111 (2015).

4. Bohigas, O., Tomosovic, S. \& Ullmo, D. Manifestations of classical phase space structures in quantum mechanics. Phys. Rep. 223, 43-133 (1993).

5. Chaudhury, S. et al. Quantum signatures of chaos in a kicked top. Nature 461, 768-771 (2009)

6. Steck, D. A., Oskay, W. H. \& Raizen, M. G. Observation of chaos-assisted tunneling between islands of stability. Science 293, 274-278 (2001).

7. Nöckel, J. U. \& Stone, A. D. Ray and wave chaos in asymmetric resonant optical cavities. Nature 385, 45-47 (1997).

8. Gmachl, C. et al. High-power directional emission from microlasers with chaotic resonators. Science 280, 1556-1564 (1998).

9. Dembowski, C. et al. First experimental evidence for chaos-assisted tunneling in a microwave annular billiard. Phys. Rev. Lett. 84, 867-870 (2000).

10. Podolskiy, V. A. \& Narimanov, E. E. Semiclassical description of chaos-assisted tunneling. Phys. Rev. Lett. 91, 263601 (2003).

11. Podolskiy, V. A. \& Narimanov, E. E. Chaos-assisted tunneling in dielectric microcavities. Opt. Lett. 30, 474-476 (2005).

12. Shinohara, S. et al. Chaos-assisted directional light emission from microcavity lasers. Phys. Rev. Lett. 104, 163902 (2010).

13. Kwak, H. et al. Nonlinear resonance-assisted tunneling induced by microcavity deformation. Sci. Rep. 5, 9010 (2015).

14. Gehler, S. et al. Experimental observation of resonance-assisted tunneling. Phys. Rev. Lett. 115, 104101 (2015).

15. Liu, S. et al. Transporting the optical chirality through the dynamical barriers in optical microcavities. Laser Photonics Rev. 12, 1800027 (2018).

16. Jiang, $X$. F. et al. Chaos-assisted broadband momentum transformation in optical microresonators. Science 358, 344-347 (2017).

17. Löck, S. et al. Regular-to-chaotic tunneling rates: from the quantum to the semiclassical regime. Phys. Rev. Lett. 104, 114101 (2010).

18. Song, Q. H. et al. Channeling chaotic rays into waveguides for efficient collection of microcavity emission. Phys. Rev. Lett. 108, 243902 (2012)

19. Kim, M. W. et al. Chaos-assisted tunneling in a deformed microcavity laser. Opt. Express 21, 32508-32515 (2013).

20. Preu, S. et al. Directional emission of dielectric disks with a finite scatterer in the THz regime. Opt. Express 21, 16370-16380 (2013).

21. Li, J. et al. Sideband spectroscopy and dispersion measurement in microcavities. Opt. Express 20, 26337-26344 (2012).

22. Lu, X. Y. et al. Universal frequency engineering tool for microcavity nonlinear optics: multiple selective mode splitting of whispering-gallery resonances. Photonics Res. 8, 1676-1686 (2020).

23. Yi, C. H., Kullig, J. \& Wiersig, J. Pair of exceptional points in a microdisk cavity under an extremely weak deformation. Phys. Rev. Lett. 120, 093902 (2018).
24. Shim, J. B. et al. Uncertainty-limited turnstile transport in deformed microcavities. Phys. Rev. Lett. 100, 174102 (2008).

25. Ge, L. Quantum chaos in optical microcavities: a broadband application. EPL 123, 64001 (2018)

26. Chen, L. K. et al. Regular-orbit-engineered chaotic photon transport in mixedphase space. Phys. Rev. Lett. 123, 173903 (2019).

27. Miao, P. et al. Orbital angular momentum microlaser. Science 353, 464-467 (2016).

28. Cognée, K. G. et al. Cooperative interactions between nano-antennas in a high-Q cavity for unidirectional light sources. Light. Sci. Appl. 8, 115 (2019).

29. Cao, Q. T., Chen, Y. L. \& Xiao, Y. F. Chiral emission and Purcell enhancement in a hybrid plasmonic-photonic microresonator. Light.: Sci. Appl. 9, 4 (2020).

30. Chen, W. J. et al. Exceptional points enhance sensing in an optical microcavity. Nature 548, 192-196 (2017).

31. Jiang, X. F. \& Yang, L. Optothermal dynamics in whispering-gallery microresonators. Light. Sci. Appl. 9, 24 (2020).

32. Huang, C. et al. Ultrafast control of vortex microlasers. Science $\mathbf{3 6 7}, \mathbf{1 0 1 8 - 1 0 2 1}$ (2020).

33. Breunig, I. et al. Whispering gallery modes at the rim of an axisymmetric optical resonator: analytical versus numerical description and comparison with experiment. Opt. Express 21, 30683-30692 (2013).

34. Schunk, $G$. et al. Identifying modes of large whispering-gallery mode resonators from the spectrum and emission pattern. Opt. Express 22, 30795-30806 (2014).

35. Gmachl, C. et al. Kolmogorov-Arnold-Moser transition and laser action on scar modes in semiconductor diode lasers with deformed resonators. Opt. Lett. $\mathbf{2 7}$ 824-826 (2002).

36. Fan, L. R. et al. Real-time observation and control of optical chaos. Sci. Adv. 7, eabc8448 (2021).

37. Schwefel, H. G. L. et al. Dramatic shape sensitivity of directional emission patterns from similarly deformed cylindrical polymer lasers. J. Optical Soc. Am. B 21, 923-934 (2004).

38. Wiersig, J. \& Hentschel, M. Combining directional light output and ultralow loss in deformed microdisks. Phys. Rev. Lett. 100, 033901 (2008).

39. Sridhar, S., Hogenboom, D. O. \& Willemsen, B. A. Microwave experiments on chaotic billiards. J. Stat. Phys. 68, 239-258 (1992).

40. Gokirmak, A. et al. Scanned perturbation technique for imaging electromagnetic standing wave patterns of microwave cavities. Rev. Sci. Instrum. 69 3410-3417 (1998)

41. Almeida, V. R. et al. All-optical control of light on a silicon chip. Nature $\mathbf{4 3 1}$ 1081-1084 (2004).

42. Gensty, T. et al. Wave chaos in real-world vertical-cavity surface-emitting lasers. Phys. Rev. Lett. 94, 233901 (2005).

43. Aldaya, I. et al. Nonlinear carrier dynamics in silicon nano-waveguides. Optica 4, 1219-1227 (2017)

44. Ge, L. et al. Extreme output sensitivity to subwavelength boundary deformation in microcavities. Phys. Rev. A 87, 023833 (2013).

45. Ge, L. \& Feng, L. Optical-reciprocity-induced symmetry in photonic heterostructures and its manifestation in scattering PT-symmetry breaking. Phys. Rev A 94, 043836 (2016).

46. Haus, H. A. Waves and Fields in Optoelectronics. (Englewood: Prentice-Hall, 1984).

47. Landau, L. D. \& Lifshitz, E. M. Electrodynamics of Continuous Media. (Oxford: Pergamon Press, 1960).

48. Qian, Y. J. et al. Observation of a manifold in the chaotic phase space of an asymmetric optical microcavity. Photonics Res. 9, 364-369 (2021).

49. Hoover, W. G. Time Reversibility, Computer Simulation, and Chaos. (Singapore: World Scientific, 1999).

50. Altmann, E. G., Portela, J. S. E. \& Tél, T. Leaking chaotic systems. Rev. Mod. Phys. 85, 869-918 (2013)

51. Zhang, Z. F. et al. Tunable topological charge vortex microlaser. Science $\mathbf{3 6 8}$ 760-763 (2020).

52. Zhang, Z. F. et al. Elimination of spatial hole burning in microlasers for stability and efficiency enhancement. ACS Photonics 5, 3016-3022 (2018).

53. Wang, B. C. et al. Towards high-power, high-coherence, integrated photonic mmWave platform with microcavity solitons. Light Sci. Appl. 10, 4 (2021).

54. Chen, H. J. et al. Chaos-assisted two-octave-spanning microcombs. Nat. Commun. 11, 2336 (2020) 